\title{
The accuracy of brackets placement in direct bonding technique: a comparison between the pole-like bracket positioning gauge and the star-like bracket positioning gauge
}

\section{Lahcen Ousehal, Laila Lazrak}

Department of Orthodontics, Faculty of Dentistry, Casablanca, Morocco.

Email: lahcen2228@yahoo.fr

Received 13 April 2011; revised 24 June 2011; accepted 3 August 2011.

\begin{abstract}
The accuracy of brackets placement is a key factor in successful orthodontic therapy. An in vitro study was conducted in ten models from a natural maxillary teeth model in order to compare the accuracy of brackets placement between two direct bonding instruments: the pole-like Bracket Positioning Gauge and the star-like Bracket Positioning Gauge. Our results have shown that: the star-like Bracket Positioning Gauge is more precise in placing brackets vertically, whereas the pole-like Bracket Positioning Gauge allows a better angulation of the bracket. Considering each tooth separately, there is no statistically significant difference between the two positioning gauges, except that the star-like gauge gives better results in bracket's height for the second premolar and the canine, whereas the pole-like gauge allows for a better positioning and a better vertical angulation of the brackets for the lateral incisor. No statistically significant difference was found between the two gauges on the mesiodistal position. Overall, the star-like gauge showed a better accuracy in positioning brackets.
\end{abstract}

Keywords: Direct Bonding; Pole-Like Gauge; Star-Like Gauge

\section{INTRODUCTION}

Good placement of orthodontic brackets guarantees a successful mechanical treatment [1]. Direct bonding is the technique that is most used by orthodontists in their daily practice [2].

Two positioning instruments are available to the practitioner, the pole like gauge and the star like gauge also known as Boone gauge.

The question that arises is which of these two instruments is more accurate in terms of positioning orthodon- tic brackets.

In literature, there are no studies comparing the pole like gauge and the star like gauge in bonding brackets. Our work has taken as objective to compare the accuracy of orthodontic bracket placement between two instruments of direct bonding: the star like and the pole like gauges.

\section{MATERIALS AND METHODS}

An experiment with a set of healthy natural teeth taken from 15 to 25 was conducted using a mold. Ten extra hard plaster models were made from the original model using alginate.

The natural teeth model was used for the ideal brackets' placement.

Eleven sets of Edgwise standard metal brackets 022 . 028 were used for the study.

The plaster models were mounted on mannequins to be close to the clinical conditions during the bonding of brackets.

The right hemi arch was reserved for direct bonding using the pole like gauge, and the left hemi arch was used for direct bonding using the star like gauge.

Four experienced orthodontists have realized the bonding of brackets following the rules of correct bonding which are: the bracket axis should be parallel to the long axis of the tooth, the bracket should be centered in the crown, the height of bonding should be set to 4, 4.5 and $3.5 \mathrm{~mm}$ for the central incisors, bicuspids, canines and lateral incisors respectively.

The bonding material used is 3M TRANSBOND self cured composite resin.

The natural teeth from the reference model have been uncovered upwards to see their roots. This facilitates the alignment of the vertical axis of the tooth. Only one practitioner positioned the brackets ideally on the reference model, held by hand, in order to control the bonding in three dimensions (Figure 1). 


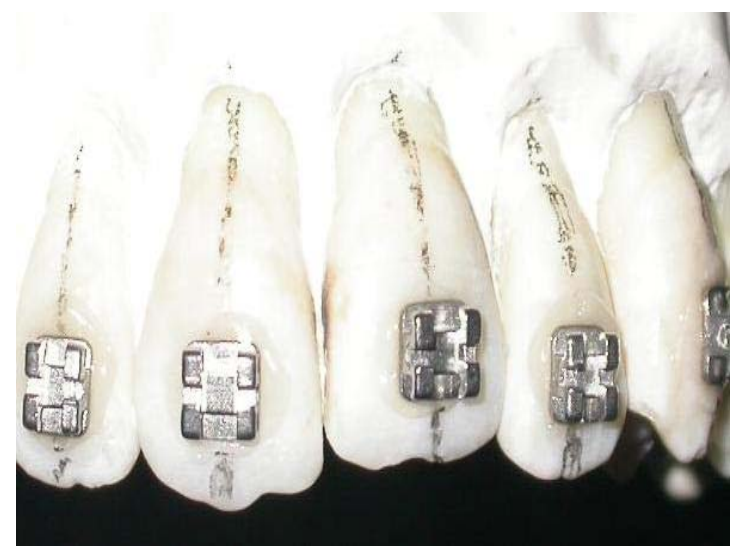

Figure 1. Positioning the brackets on the reference model.

A metal ruler and a protractor scale $0.5 \mathrm{~mm}$ were used for linear and angular measures.

Standardized photographs of each bonded tooth of ten plaster models and the reference model have been taken with an analog camera (III Yascika dental eye.) The camera was fixed on a tripod allowing for an effective control of the vertical distance from which the images were taken.

The expansion used is $1 / 1$ and the photographs were enlarged ten times to facilitate measurements.

\section{Methods}

The contour of the crown, the vertical center line and the horizontal center line of the bracket are drawn on a tracing paper laid over the photo of each tooth.

Each paper is laid over the picture of the natural tooth equivalent in the reference model.

Two linear measures and one angular measure were calculated between the ideal group and the tested group (Figure 2).

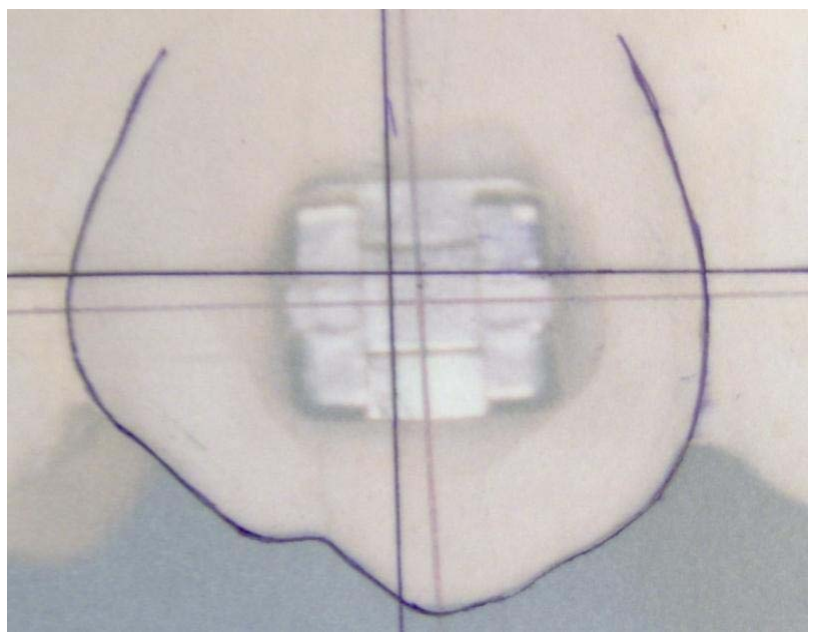

Figure 2. Tracing paper overlaid on the photo of the dog stuck by the direct method showing the different steps.
The height of the bracket: A zero value was assigned to the bracket that bonded perfectly to the center.

A negative value was assigned when the vertical gap between the bracket bonded with the gauge and the ideal bracket is occlusal. Conversely, if the gap is cervical, a positive value is assigned.

Mesio distal inclination: The horizontal distance between the center of the ideal bracket and that of the tested bracket was measured to assess the mesio distal gap of the bracket. A positive value was assigned when the elevation of the tested bracket is mesial compared to that of the ideal bracket, and a negative value is assigned when the gap is distal.

Angulation: It is determined by the angle between the horizontal center line of the ideal bracket and that of the tested bracket.

This measure is positive when the center line of the tested bracket is cervical relative to the ideal bracket and negative in the opposite case.

Statistical analysis: A statistical software (EPI-INFO) was used in order to collect and analyze all the measurements. The Student $\mathrm{T}$ test was used for statistical comparison of the results. The measurements were repeated for reproducibility and reliability of results. A paired $t$ test has been realized and the $t$ test is less than the calculated t-test table, we can say there is no significant difference between the 2 measurements.

\section{RESULTS}

Tables 1 and 2 show the averages and standard deviation for each group of teeth of the three measures: vertical, mesiodistal, and angular.

Vertical positioning: The comparison between the group of teeth bonded with the star-like gauge and the polelike gauge shows a very significant difference $(\mathrm{P}=0.006)$ for the second premolar and a significant difference for the canine and the lateral incisor (Table 3).

The star-like gauge is more accurate for the second premolar and the canine. The pole-like gauge is more accurate for the lateral incisor.

Mesiodistal positioning: The comparison between the group of teeth bonded with the star-like gauge and the pole-like gauge shows a significant difference for the first premolar and the lateral incisor (Table 4). Indeed, the mesiodistal gap are opposed relatively to the center in the two groups. For the first premolar, we have a mesial gap of $0.32 \mathrm{~mm}$ on average for the group of teeth that bonded with the pole-like gauge, and a distal gap of 0 , $46 \mathrm{~mm}$ for the group of teeth that bonded with the starlike gauge.

Similarly, for the lateral incisor, we have a mesial gap of $0.42 \mathrm{~mm}$ with the pole-like gauge, and a distal gap of $0.38 \mathrm{~mm}$ with the star-like gauge.

Both instruments lead to mesiodistal placement errors. 
Inclination of the bracket: The angular gap of the bracket shows a significant difference only for the lateral in- cisor (Table 5). The pole-like gauge is more accurate in this case.

Table 1. Mean values and standard deviation (STDV) of the vertical, the mesiodistal and the angular brackets' position on the left hemi arch.

\begin{tabular}{cccccccccccc}
\hline & \multicolumn{2}{c}{25} & \multicolumn{2}{c}{24} & \multicolumn{2}{c}{23} & & & & 22 & \\
Teeth & Mean & STDV & Mean & STDV & Mean & STDV & Mean & STDV & Mean & STDV \\
\hline Height (mm) & -0.43 & 0.129 & -0.46 & 0.314 & -0.29 & 0.207 & -0.38 & 0.109 & -0.31 & 0.217 \\
M-D (mm) & 0.10 & 0.237 & -0.45 & 0.228 & -0.03 & 0.262 & -0.38 & 0.230 & 0.01 & 0.242 \\
$\AA$ A (degrees) & -0.32 & 0.228 & -0.12 & 0.177 & -0.17 & 0.130 & -0.30 & 0.146 & -0.24 & 0.133 \\
\hline
\end{tabular}

Table 2. Mean values and standard deviation of the vertical, the mesiodistal and the angular brackets' position on the right hemi arch.

\begin{tabular}{cccccccccccc}
\hline & \multicolumn{3}{c}{15} & \multicolumn{2}{c}{14} & \multicolumn{2}{c}{13} & & 12 & & \multicolumn{2}{c}{11} \\
\hline Teeth & Mean & SDTV & Mean & SDTV & Mean & SDTV & Mean & SDTV & Mean & SDTV \\
\hline Height (mm) & -1.02 & 0.430 & -0.38 & 0.204 & -0.47 & 0.440 & 0.26 & 0.214 & -0.005 & 0.281 \\
M-D (mm) & -0.04 & 0.205 & 0.32 & 0.155 & 0.32 & 0.216 & 0.42 & 0.116 & 0.03 & 0.155 \\
$\AA$ (degrees) & -0.23 & 0.131 & -0.17 & 0.056 & -0.21 & 0.228 & 0.16 & 0.168 & -0.05 & 0.215 \\
\hline
\end{tabular}

Table 3. Comparison of the vertical gaps between the star-like and the pole-like gauges.

\begin{tabular}{|c|c|c|c|c|c|}
\hline \multirow{2}{*}{ Teeth } & \multicolumn{2}{|c|}{ Pole-like gauge } & \multicolumn{2}{|c|}{ Star-like gauge } & \multirow{2}{*}{ P-value } \\
\hline & Mean (mm) & SDTV & Mean (mm) & SDTV & \\
\hline $2^{\text {nd }} P M$ & -1.020 & 0.430 & -0.435 & 0.129 & $0.006^{* *}(\mathrm{~S})$ \\
\hline $1^{\text {st }} \mathrm{PM}$ & -0.315 & 0.204 & -0.460 & 0.228 & 0.54 \\
\hline Canine & -0.470 & 0.440 & -0.030 & 0.262 & $0.05 *(S)$ \\
\hline Lateral incisor & 0.260 & 0.214 & -0.380 & 0.109 & $0.04 *(S)$ \\
\hline Central incisor & -0.005 & 0.281 & -0.315 & 0.217 & 0.87 \\
\hline
\end{tabular}

Table 4. Comparison of the mesiodistal gap between the star-like and the pole-like gauges.

\begin{tabular}{|c|c|c|c|c|c|}
\hline \multirow{2}{*}{ Tooth } & \multicolumn{2}{|c|}{ Pole-like gauge } & \multicolumn{2}{|c|}{ Star-like gauge } & \multirow{2}{*}{ P-value } \\
\hline & Mean (mm) & SDTV & Mean (mm) & SDTV & \\
\hline $2^{\text {nd }} P M$ & -0.040 & 0.205 & 0.105 & 0.237 & 0.66 \\
\hline $\mathbf{1}^{\text {st }} \mathbf{P M}$ & 0.320 & 0.155 & -0.460 & 0.428 & $0.04 *(S)$ \\
\hline Canine & 0.328 & 0.216 & -0.030 & 0.262 & 0.43 \\
\hline Lateral incisor & 0.425 & 0.116 & -0.380 & 0.230 & $0.03 *(S)$ \\
\hline Central incisor & -0.030 & 0.155 & 0.010 & 0.242 & 0.74 \\
\hline
\end{tabular}

Table 5. Comparison of the inclination gaps between the star-like and the pole-like gauges.

\begin{tabular}{cccccc}
\hline \multirow{2}{*}{ Teeth } & \multicolumn{2}{c}{ Pole-like gauge } & \multicolumn{2}{c}{ Star-like gauge } & \multirow{2}{*}{ P-value } \\
\cline { 2 - 5 } & Mean (degrees) & SDTV & Mean (degrees) & SDTV & 0.73 \\
\hline $2^{\text {nd }} \mathrm{PM}$ & -0.235 & 0.131 & -0.320 & 0.258 & 0.84 \\
$1^{\text {st }} \mathrm{PM}$ & -0.173 & 0.056 & -0.125 & 0.177 & 0.86 \\
Canine & -0.210 & 0.228 & -0.170 & 0.130 & $0.04^{*}(\mathrm{~S})$ \\
Latéral Incisor & 0.160 & 0.168 & -0.305 & 0.146 & $0.05^{*}(\mathrm{~S})$ \\
Central Incisor & -0.050 & 0.215 & -0.240 & 0.133 & \\
\hline
\end{tabular}




\section{DISCUSSION}

The objective of our work was to compare two instruments of direct bonding of brackets that are commonly used by orthodontists: the star-like gauge and the polelike gauge.

We conducted our study on the maxillary arch because we believe that it is the arch in which the placement of brackets in direct bonding is the most difficult.

We have also reproduced (in part) the clinical conditions by mounting the models on mannequins and using the same adhesive product used in clinical practice.

The choice of experienced orthodontists in our study reduces errors related to the competence of the practitioner.

Similarly, the use of multiple practitioners is more realistic in that it avoids ending up with the same bonding errors related to a single practitioner.

The comparison between the two methods showed that the two instruments cause overall the same placement errors, but that we have more accuracy with the star-like gauge, especially for the vertical placement of brackets.

The photographic assessment is a reliable way to study the position of the bracket, provided the same protocol and the same parameters are followed.

In our study, we used a system of rigid fixation and the same magnification for all pictures. This greatly reduces errors related to distortions of the photographic image.

The enlargement of the photographs was performed only for conveniently reading the various measures.

The pole-like gauge showed less accuracy in the vertical placement of bracket; this can be explained by its nature that allows it to be inclined too occlusally or too cervically, depending on the practitioner's position relative to the bonding tooth.

Our results showed a greater inclination of the bracket with the pole-like gauge on the lateral incisor.

The orthodontic literature has focused mainly on comparing the accuracy of brackets placement between direct and indirect bonding [3-7]. No study has taken before this one the objective of comparing bonding with the pole-like gauge and the star-like gauge.

BOHN CHAN KOO [8] conducted an in vitro study comparing the accuracy of bracket placement between direct and indirect technique.

For this, 19 sets of models of the same occlusion were divided into two groups, one for direct bonding (9 models), the second for indirect bonding (9 models).

A model has been set aside for ideal bonding (comparison model). The bonding was done by nine experienced orthodontists.

Direct bonding has been achieved by the star-like gauge similarly to our work, comparing the accuracy bonding on the basis of three measurements: vertical, angular, and mesiodistal using enlarged photographs of each tooth.

The author concluded that there is a gap between indirect bonding and ideal bonding. This is consistent with our results on the direct bonding.

He also noted that for the maxillary second premolar, indirect bonding is more accurate, whereas for the maxillary lateral incisor, direct bonding was better for the inclination of the bracket.

In our work we found that the pole-like gauge allows precise angle of the bracket for the lateral incisor, and we recorded largest gaps for the maxillary second premolar.

Similar results have been presented by BALUT [2].

In his work, he studied the accuracy of direct bonding on models having the same malocclusion. He found that the average inclination was $5.54^{\circ}$ and $0.34 \mathrm{~mm}$ for the height.

Another study was conducted by Aguirre, KING and WALDRON [9]. It is a clinical evaluation done to determine the advantages and disadvantages of both techniques.

It focuses on 11 patients, 206 bonded brackets, and 189 measured brackets. The maxillary and mandibular arches are divided into hemi-arches, randomly bonding one side according to a technique different to the reverse side.

In direct technique [10], the brackets are positioned with the star-like gauge (gauge Boone UNITEK).

To assess the placement of brackets, photographs of each bonded tooth are taken in similar ways and are compared with the corresponding tooth on the other he$\mathrm{mi}$ arch. To get accurate conclusions, the authors analyzed in turn:

- Linear measurements.

- Angular values.

Concerning the linear measurements (height placement), the results showed that neither techniques was $100 \%$ accurate. If we compare direct and indirect methods, no significant difference in the maxillary arch was found, except for the canines for which brackets bonded using the indirect technique are placed near the ideal height.

The angular measurements have also shown that both methods fail to place ideally brackets with great variability. Apparently, the practitioner has more difficulty in judging the angles relative to height. In comparing direct and indirect technique in the upper arch, there is no significant difference except for the canines with an advantage with the indirect technique, which led to an angle closer to the ideal angle of 90 . The results of this study should perhaps be brought into perspective, because all the brackets are bonded by the same practitioner. Indeed, these results may reflect the clinical skill of a particular practitioner rather than the difference between the two techniques. 


\section{CONCLUSIONS}

With the advent of straight-wire techniques, the positioning of brackets has taken a significant role in the success of orthodontic treatment.

The placement of orthodontic brackets is a difficult but crucial, even in straight wire techniques, which use pre-informed brackets.

In this case, any small placement error leads all the brackets data to be distorted proportionally to the placement error.

In everyday practice, most orthodontists use two types of gauge for brackets placement: the star-like gauge and the pole-like gauge.

Our study revealed by comparing these two types of gauges that:

- The star-like gauge provides better placement of the vertical bracket, whereas the pole-like gauge allows for a better angle of the bracket.

- No statistically significant difference between these two gauges has been highlighted for the mesiodistal position.

\section{REFERENCES}

[1] Andrews, L.F. (1976) The straight-wire appliance. Explained and compared. Journal of Clinical Orthodontics, 10, 174-195.

[2] Balut, N., Klapper, L., Sandrik, J. and Bowman, D. (1992) Variations in bracket placement in the preadjusted orthodontic appliances. American Journal of Orthodontics and Dentofacial Orthopedics, 102, 62-67. doi:10.1016/0889-5406(92)70015-3
[3] Shpack, N. (2007) Bracket placement in lingual vs labial systems and direct vs indirect bonding. Angle Orthodontist, 77, 509-517. doi:10.2319/0003-3219(2007)077[0509:BPILVL]2.0.CO ;2

[4] Hodge, T.M. (2004) A randomized clinical trial comparing the accuracy of direct versus indirect bracket placement. Journal of Orthodontics, 31, 132-137. doi:10.1179/146531204225020427

[5] Wiechman, D. (2003) Customized brackets and Archwire for lingual orthodontic treatment. American Journal of Orthodontics and Dentofacial Orthopedics, 124, 593599. doi:10.1016/j.ajodo.2003.08.008

[6] Joiner, M. (2010) In-house precision bracket placement with the indirect bonding technique. American Journal of Orthodontics and Dentofacial Orthopedics, 137, 850-854. doi:10.1016/j.ajodo.2009.04.023

[7] Thomas, D.S., Salome, N., Hatch, J. and Rugh, J. (2007) Practice-based comparison of direct and indirect bonding. American Journal of Orthodontics and Dentofacial Orthopedics, 132, 738-742.

doi:10.1016/j.ajodo.2006.01.037

[8] Koo, B.C. (1999) Comparison of the accuracy of brackets placement between direct and indirect bonding techniques. American Journal of Orthodontics and Dentofacial Orthopedics, 116, 346-351. doi:10.1016/S0889-5406(99)70248-9

[9] Aguirre, M., King, G. and Waldron, J. (1982) Assessment of bracket placement and bond strength when comparing direct bonding to indirect bonding. American Journal of Orthodontics and Dentofacial Orthopedics, 82, 269-276.

[10] Nassib, B. and Klapper, L. (1992) Variations in bracket placement in the preadjusted orthodontic appliance. American Journal of Orthodontics and Dentofacial Orthopedics, 102, 62-67. doi:10.1016/0889-5406(92)70015-3 